\title{
Robust spectral phase reconstruction of time-frequency entangled bi-photon states
}

\author{
Ilaria Gianani $\odot^{*}$ \\ Dipartimento di Scienze, Università degli Studi Roma Tre, Via della Vasca Navale 84, 00146 Rome, Italy \\ and Dipartimento di Fisica, Sapienza Università di Roma, Piazzale Aldo Moro 5, 00185 Rome, Italy
}

(Received 25 January 2019; published 10 December 2019)

\begin{abstract}
Exploitation of time-frequency properties of spontaneous parametric down conversion photon pairs has recently found application in many endeavors. Complete characterization and control over the states in this degree of freedom is of paramount importance for the development of optical quantum technologies. This is achieved by accessing information both on the joint spectral amplitude and the joint spectral phase. Here we propose a scheme based on the MICE algorithm, which aims at reconstructing the joint spectral phase by adopting a multishear approach, making the technique suitable for noisy environments. We report on simulations for the phase reconstruction and propose an experiment using a Franson-modified interferometer.
\end{abstract}

DOI: 10.1103/PhysRevResearch.1.033165

\section{INTRODUCTION}

Spectral-temporal properties are among the most reliable and robust choices for encoding information for photonics quantum technologies. Being an internal degree of freedom, these are suitable for long-distance communications and allow for propagation through long-distance fibers without affecting the quantum state [1]. Applications exploiting time-frequency encoding range from quantum key distribution protocols [2-5] and clock synchronization [6] to quantum communications [7], all of which make use of frequency-correlated two-photon states.

The most common technique to generate such pairs are nonlinear processes, such as spontaneous parametric down conversion (SPDC) and four-wave mixing, in which the spectral-temporal properties are dictated by the shape of the pump as well as the material through its phase-matching function. Tailoring the pump and choosing the appropriate dispersion allow for diverse capacities in shaping spectrally broad two-photon states [8-11]. Quantum technologies demand that the information carriers be prepared in fiducial states at the beginning, as a key requirement for the correct operation of any protocol. The variegate structure of time-frequency modes is at the same time what grants its advantages but poses some critical challenges in its characterization. Ultrafast pulsed modes-exactly like their classical counterparts-vary too quickly to be characterized in the time domain. To characterize them in the frequency domain, there is a need to access both their spectral amplitude and spectral phase, as both affect the time profile and can carry signatures of frequency correlations. Measuring the

\footnotetext{
*ilaria.gianani@uniroma3.it

Published by the American Physical Society under the terms of the Creative Commons Attribution 4.0 International license. Further distribution of this work must maintain attribution to the author(s) and the published article's title, journal citation, and DOI.
}

joint spectral amplitude (JSA) is now a commonly addressed task [12-14]; however the measurement of the joint spectral phase (JSP) has only recently been tackled. This has been achieved by performing quantum state tomography on the bi-photon state $[15,16]$, by extending what is normally applied to discrete systems, e.g., polarization, or by stimulated emission [17].

An alternative route relies on classical ultrafast metrology techniques, which have been extensively developed following the need to characterize femtosecond and attosecond pulses [18]. Approaches in this direction have been recently proposed with a modified XFROG in Ref. [19], and in Ref. [20], where the self-reference classical metrology technique SPIDER [21] has been adapted to the heralded measurement of photon pair phases. SPIDER reconstructs the spectral phase by retrieving the interferometric phase between two frequency-sheared copies of an unknown pulse. The extraction algorithm is quite simple and is based on the integration of the interferometric phase. In the last few decades many different implementations of SPIDER have been developed to address increasing degrees of pulse complexity [22-25]. In particular multishear techniques such as SEA-CAR SPIDER [26-28] have provided a very robust tool for the reconstruction of broadband pulses with high spectral complexity. In all its implementations SPIDER is a referenced technique, where the reference is either the pulse itself or, with a slight modification, a known external field. More recently a new algorithm, MICE [29], which relies as well on multishear techniques, has been developed. Contrary to the standard SPIDER extraction algorithm, MICE allows for the mutual characterization of multiple unknown fields at the same time. Due to the redundancies achieved via the multishear arrangement, MICE performs extremely well even under very stringent noise conditions. This technique has proven to be extremely versatile in the classical regime and it has been employed for the reconstruction of spectral phases of complex pulses in the visible-near-IR regime [30], for wave front reconstruction [29], for the spatial characterization of high-harmonic sources [31], and for digital holography microscopy [32]. 
Here we propose how to interface MICE with the specific needs of quantum light detection. Merging together the two tools demands devising a detection scheme for which MICE can be adopted and feed back the requirements of the detection to the implementation of MICE. In order to do so, the measurement strategy relies on the use of a modified Franson interferometer $[33,34]$ which is commonly adopted in quantum optics but has no equivalent in classical metrology. The modified Franson scheme allows us to observe genuine timebin entanglement without relying on time-resolved detection. This is a necessary condition to obtain coincidences which are dependent on the bi-photon spectral phase to be extracted. Simulations show that due to the redundancy provided by the multishear approach, the technique works reliably even with moderate signal intensities.

\section{QUANTUM MICE}

MICE is a classical metrology technique which uses an iterative algorithm to simultaneously reconstruct multiple unknown fields $E_{i}$ depending on a set of parameters $\gamma$ without the need of an external known reference. This is made possible by means of a multishear measurement strategy, in which multiple shears must be used to scan the fields along each parameter, and the number of fields to reconstruct must be lower than the number of shears used for each dimension. This is sufficient to guarantee enough redundancy, which makes the technique particularly robust against noise. Given two fields $E_{1}(\gamma)$ and $E_{2}(\gamma)$, MICE relies on the minimization of the error with respect to each field [29]:

$$
\mathcal{E}=\sum_{j, k, l}\left|\mathrm{AC}_{j-k, j-l}^{\text {meas }}-E_{1}\left(\gamma_{j}-\Gamma_{k}\right) E_{2}^{*}\left(\gamma_{j}-\Gamma_{l}\right)\right|^{2},
$$

where $\mathrm{AC}^{\text {meas }}$ is the measured interferometric product between the two fields, obtained as the sideband of the Fourier transform of $I_{k, l}=\left|E_{1}\left(\gamma-\Gamma_{k}\right)+E_{2}\left(\gamma-\Gamma_{l}\right)\right|^{2}$. Measuring the bi-photon spectral phase requires implementing interferometric schemes, which typically demand long accumulation times to achieve good signal levels. Given its robustness against noise, using MICE grants a solution to this, becoming the preferable choice for such an endeavor. This is conditioned on properly choosing an arrangement whose measurement outcome obeys the behavior described above.

Consider the modified version of the interferometric scheme proposed by Cabello et al. [33], as depicted in Fig. 1. The original motivation of this scheme lies in easing some technical requirements of Franson's original idea [35] for the generation of time-bin entanglement. A photon pair is generated via SPDC; both the signal and idler photons can undertake either a short $|S\rangle$ or a long $|L\rangle$ path before being detected with a frequency-resolved measurement. This scheme has been proved to generate time-bin entanglement between the short and long paths without relying on time-resolved detection [33]. In order to make it suitable for our purposes, two further modifications need to be introduced: frequencyresolved detection is adopted; independent frequency shears are inserted on the $S_{s}$ and $L_{i}$ path: the signal will be sheared only when taking the short path, the idler only when taking the long one. Shearing can be performed by means of electrooptic modulators (EOMs) as proposed and demonstrated by

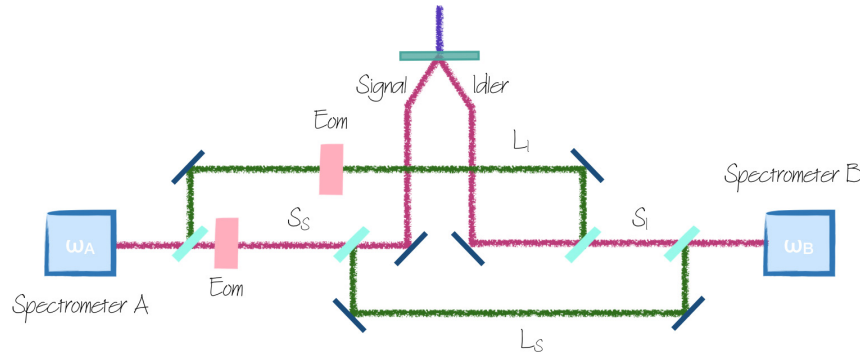

FIG. 1. Proposed interferometric scheme. A photon pair produced via SPDC enters a modified Franson interferometer where each photon can undertake either a long (L) or short (S) path. When the signal (idler) photon passes through the short (long) path it is subject to a frequency shear given by the EOM. A frequencyresolved coincidence counting measurement is then performed.

[20,36]. This is preferable to nonlinear optical shearing, as we work in the single-photon regime. Since we adopt a multishear approach, both the shears have to be scanned independently through multiple values, so that for each shear $\left(\Omega_{1, k}\right)$ on $S_{s}$, the shear $\left(\Omega_{2, l}\right)$ on $L_{i}$ scans along the idler dimension of the joint spectral wave function. In the most general case, MICE is not bounded to work with fields having the same spectral support, if the shears are chosen so that the interferograms will completely cover the fields along every dimension. In fact, the phase will only be retrieved in the zones covered by the interference. At the same time the interferograms given by two subsequent shears need to overlap; otherwise they cannot make use of the redundancy. If the fields interfering have the same support, the sole purpose of the multiple shears is to grant the redundancy; hence they can be as small as allowed by the detection resolution. The state entering the interferometer will be given by [37]

$$
\Psi\left(\omega_{s}, \omega_{i}\right)=\int d \omega_{s} d \omega_{i} A\left(\omega_{s}, \omega_{i}\right) a_{s}^{\dagger}\left(\omega_{s}\right) a_{i}^{\dagger}\left(\omega_{i}\right)|0\rangle|0\rangle,
$$

where $A\left(\omega_{s}, \omega_{i}\right)=\operatorname{JSA}\left(\omega_{s}, \omega_{i}\right) e^{i \operatorname{SP}\left(\omega_{s}, \omega_{i}\right)}$ is the wave function of the bi-photon state. As the photon pair goes through the interferometer, the output state $\tilde{\Psi}$ will be transformed into

$$
\begin{aligned}
& \tilde{\Psi}\left(\omega_{s}, \omega_{i}, \Omega_{1, k}, \Omega_{2, l}, \tau\right) \\
& =\int d \omega_{s} d \omega_{i} A\left(\omega_{s}, \omega_{i}\right) a_{s}^{\dagger}\left(\omega_{s}+\Omega_{1, k}\right) a_{i}^{\dagger}\left(\omega_{i}\right) \\
& \quad+A\left(\omega_{s}, \omega_{i}\right) a_{s}^{\dagger}\left(\omega_{s}\right) a_{i}^{\dagger}\left(\omega_{i}+\Omega_{2, l}\right) e^{i\left(\omega_{s}+\omega_{i}+\Omega_{2, l}\right) \tau}|0\rangle|0\rangle,
\end{aligned}
$$

where $\tau$ is the delay between the two paths and we have supposed performing the shear on the $L_{i}$ path after a length equal to that of the $S$ paths. We notice however that due to the modified geometry, the detector will not always measure $\omega_{s}$ or $\omega_{i}$, and that is a fundamental requirement to assure genuine time-bin entanglement between the two photons, as it allows us to automatically discard the $|S\rangle|L\rangle$ and $|L\rangle|S\rangle$ events. The measurement on $\tilde{\Psi}\left(\omega_{s}, \omega_{i}, \Omega_{1, k}, \Omega_{2, l}, \tau\right)$ resolves the frequencies $\omega_{A}$ and $\omega_{B}$ at each detection site. When both photons undertake the short path, detector $A$ will measure $\omega_{A}=\omega_{s}-\Omega_{1}$ and detector $B \omega_{B}=\omega_{i}$; when both photons take the long path, $\omega_{A}=\omega_{i}-\Omega_{2}, \omega_{B}=\omega_{s}$. 
The measured coincidence probability reads

$$
P\left(\omega_{A}, \omega_{B}\right)=\left|A\left(\omega_{A}, \omega_{B}\right)+A\left(\omega_{B}, \omega_{A}\right) e^{i\left(\omega_{A}+\omega_{B}\right) \tau}\right|^{2}
$$

which, by defining $A_{1}\left(\omega_{A}, \omega_{B}\right)=A\left(\omega_{A}, \omega_{B}\right)$ and $A_{2}\left(\omega_{B}, \omega_{A}\right)=A\left(\omega_{B}, \omega_{A}\right) e^{i\left(\omega_{A}+\omega_{B}\right) \tau}$, in terms of the signal and idler frequencies, becomes

$$
\begin{aligned}
& P\left(\omega_{s}, \omega_{i}, \Omega_{1, k}, \Omega_{2, l}\right) \\
& \quad=\left|A_{1}\left(\omega_{s}-\Omega_{1, k}, \omega_{i}\right)+A_{2}\left(\omega_{s}, \omega_{i}-\Omega_{2, l}\right)\right|^{2},
\end{aligned}
$$

which has the same structure of the interferogram $I$ between $E_{1}(\gamma)$ and $E_{2}(\gamma)$, where $\gamma$ corresponds to the set of parameters $\gamma=\omega_{s}, \omega_{i}$. As such, this can now be processed with the MICE algorithm, solving the following equations which have been obtained by minimizing the error in Eq. (1) with respect to both fields $\left(\partial \mathcal{E} / \partial A_{i}=0\right)$ :

$$
\begin{aligned}
& A_{1}\left(\omega_{i}, \omega_{j}\right)=\frac{\sum_{k, l} \mathrm{AC}_{i-k, j+l}^{\text {meas }} A_{2}^{*}\left(\omega_{i}+\Omega_{1, k}, \omega_{j}-\Omega_{2, l}\right)}{\sum_{k, l} A_{2}\left(\omega_{i}-\Omega_{1, k}, \omega_{j}+\Omega_{2, l}\right)}, \\
& A_{2}^{*}\left(\omega_{i}, \omega_{j}\right)=\frac{\sum_{k, l} \mathrm{AC}_{i+k, j-l}^{\text {meas }} A_{1}^{*}\left(\omega_{i}-\Omega_{1, k}, \omega_{j}+\Omega_{2, l}\right)}{\sum_{k, l} A_{1}\left(\omega_{i}-\Omega_{1, k}, \omega_{j}+\Omega_{2, l}\right)} .
\end{aligned}
$$

To solve this set of equations it is necessary to provide an initial guess for $A_{2}$ to obtain an initial value of $A_{1}$, which is then fed into the second equation. By iteration the two fields are retrieved. Here we do not make use of the fact that the two fields are strongly related. We can leave the structure of the MICE equations unaltered and compare the reconstructed field as means of verifying the consistency of the reconstruction. We remark that as any implementation based on SPIDER, MICE suffers form ambiguities in determining the amplitude $X\left(\omega_{s}, \omega_{i}\right)$ : the phases retrieved for both fields will be accurate, but the amplitudes will not. In order to retrieve the JSA with the setup proposed, it would be sufficient to block the $L$ arms, and perform the spectral measurement on the $S$ arms alone.

\section{NUMERICAL TESTS}

In order to test the analysis routine, we perform a bi-photon phase reconstruction on simulated data. The JSA and JSP constituting $A_{1}$ are shown in panels (a) and (b) of Fig. 2, and are those typically emitted (e.g., those in Refs. $[15,20])$. The field is sampled on a $32 \times 32$ pixel grid, covering a spectral range of $10 \mathrm{~nm}$ centered at $820 \mathrm{~nm}$ along each dimension. $A_{2}$ is obtained as a permutation between the two dimensions of $A_{1}$. The shears are then applied to both fields. Both $\Omega_{1}$ and $\Omega_{2}$ can each assume 8 different values, which leads to 64 interferograms per reconstruction. As per Fig. 2, we choose a scenario in which the correlations are present only in the phase to be retrieved and not in the JSA. Since the amplitude of the two fields is the same and the multiple shears are used only for the required redundancy, their value can be as small as dictated by the detection resolution, so in order to have eight different values, the shear on each arm will vary between -4 pixels and 3 pixels (where each pixel corresponds to $\Delta \lambda \sim 0,3 \mathrm{~nm})$.

To test against the robustness to noise in a realistic scenario, we perform different reconstructions by varying the sig-
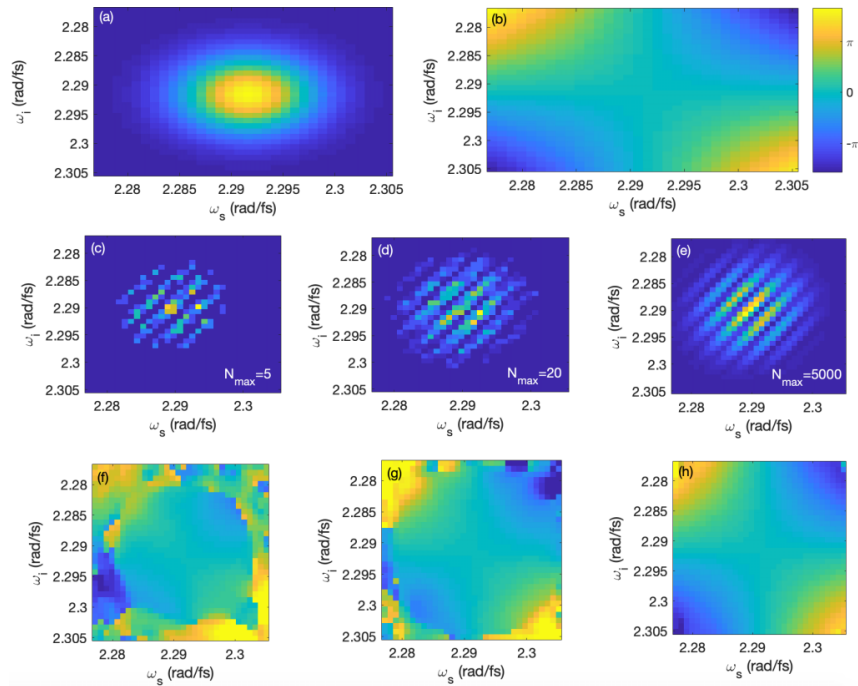

FIG. 2. Simulation results. (a) Joint spectral amplitude for $A_{1}$. (b) Joint spectral phase for $A_{1}$. (c)-(e) Interferograms obtained with 5, 20, and 5000 maximum peak coincidence counts. (f)-(h) Retrieved joint spectral phase for the three signal intensities.

nal intensity. In particular the interferograms are normalized by setting peak coincidence counts of the interferogram $N_{\text {max }}$, from 5 to 8000 coincidences. Furthermore accidental coincidences are added accordingly, given by $N_{\text {acc }}=$ $\left(N_{\max } / 0.1\right)^{2} /\left(80 \times 10^{6}\right)$, obtained considering a $10 \%$ coincidence efficiency to determine the signal intensity and a repetition rate of $80 \mathrm{MHz}$. Note that $N_{\text {acc }}$ is calculated on the maximum coincidence value and is hence overestimated. The use of $N_{\max }$ as a figure of merit was preferred to the total number of coincidences as this (for a given number of coincidences per frequency bin, set by $N_{\max }$ ) would depend solely on the spectral shape and bandwidth of the input state. The collection time as well would strongly depend on the implementation, and hence cannot be specified. The interferograms are then randomly generated with a Poissonian distribution centered at the value give by the normalization for each pixel, to which Possionian-distributed accidental coincidences are added. The interferograms are then fed to the MICE algorithm set with 20 iterations. The interferograms for $N_{\max }=5, N_{\max }=20$, and $N_{\max }=5000$ coincidence counts are shown in Fig. 2, panels (c)-(e). Panels (f)-(h) show the reconstructed JSP of $A_{1}$ for each signal intensity. The phase of $A_{2}$, which is also reconstructed, is not shown as it does not add any meaningful information.

Each reconstruction is then repeated 30 times to accumulate statistics for calculating the RMS error, weighted with the field's intensity [38], between the original and retrieved phase of $A_{1}$. The results are shown in Fig. 3. Varying the signal intensity, the error converges to its minimum of $0,0045 \mathrm{rad}$ for $N_{\max }=5000$. However even for 5 peak counts the intensity-weighted RMSE is $0.056 \mathrm{rad}$, which indicates a good agreement between the retrieved and original phase. In fact, even when the full span of the phase is not reconstructed, the low intensity does not affect the reconstruction in the portion with nonzero signal. This makes MICE an excellent tool for dealing with particularly low count rates 


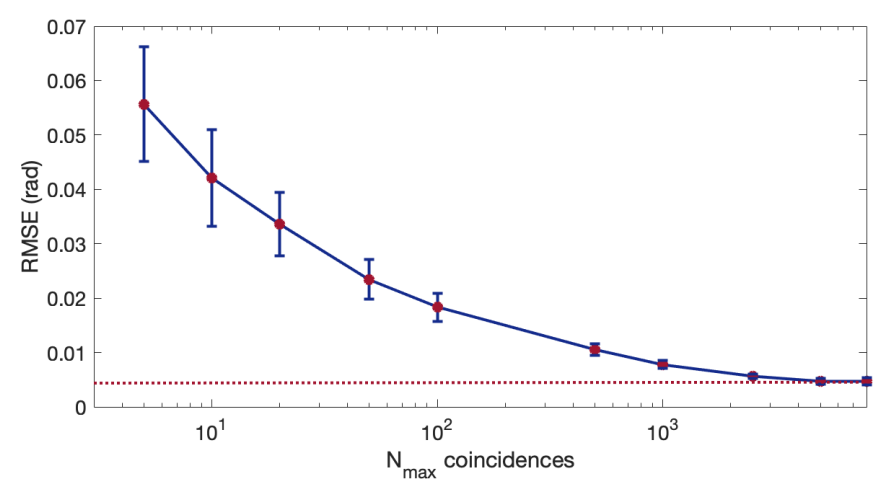

FIG. 3. RMS error between the original and retrieved phase vs the interferogram's peak coincidence counts. The error saturates at 5000 peak coincidences at $4.5 \times 10^{-3} \mathrm{rad}$.

and noisy scenarios. Shear, resolution, and signal intensity all concur in achieving a correct reconstruction and have to be tailored to the measured state, taking into account its spectral amplitude and phase complexity, which is common to every reconstruction technique in the classical domain as well. Nonetheless, with the appropriate choice of parameters, the algorithm is capable of successfully reconstructing arbitrarily complex JSPs, as demonstrated in the reconstruction in Fig. 4, where the University of Roma Tre logo has been used as the JSP. With respect to the reconstruction shown before, 32 shears were employed instead of 8; however the same resolution and spectral intensity were kept as in the previous, more realistic case. In this example the redundancy given by the multiple shears contrasts with the lack of resolution in the reconstruction of a highly structured phase, showing the
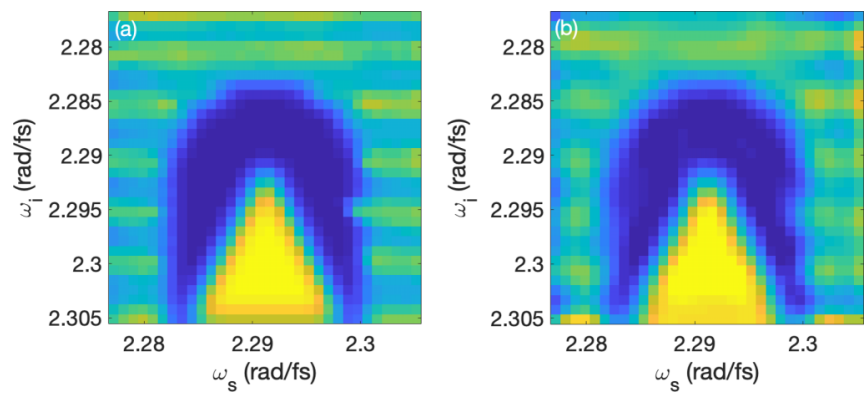

FIG. 4. JSP reconstruction of University of Roma Tre logo.

flexibility given by the interplay among the many reconstruction parameters.

Concluding, we propose of a technique which is capable of characterizing the joint spectral phase of a bi-photon state even in low-signal, noisy regimes. This takes advantage of the high redundancy granted by the multishear approach, which is implemented using a modified Franson interferometer. The robustness to noise is reflected in a rapid convergence of the RMS error to its minimum. The proposed setup presents its complexities but it has already been successfully used in many endeavors. The lack of strict signal requirements and the robustness to noise make up for these complexities, posing this technique as a possible route to obtain a complete characterization of time-frequency states.

\section{ACKNOWLEDGMENT}

The author would like to thank M. Barbieri for his helpful advice, and Ian A. Walmsley and G. Vallone for fruitful discussion.
[1] Q. Zhang, H. Takesue, S. W. Nam, C. Langrock, X. Xie, B. Baek, M. M. Fejer, and Y. Yamamoto, Opt. Express 16, 5776 (2008).

[2] J. Nunn, L. J. Wright, C. Sller, L. Zhang, I. A. Walmsley, and B. J. Smith, Opt. Express 21, 15959 (2013).

[3] J. M. Lukens, A. Dezfooliyan, C. Langrock, M. M. Fejer, D. E. Leaird, and A. M. Weiner, Phys. Rev. Lett. 112, 133602 (2014).

[4] J. Rödiger, N. Perlot, R. Mottola, R. Elschner, C. M. Weinert, O. Benson, and R. Freund, Phys. Rev. A 95, 052312 (2017).

[5] J. Mower, Z. Zhang, P. Desjardins, C. Lee, J. H. Shapiro, and D. Englund, Phys. Rev. A 87, 062322 (2013).

[6] V. Giovannetti, S. Lloyd, and L. Maccone, Nature (London) 412, 417 (2001).

[7] D. Kielpinski, J. F. Corney, and H. M. Wiseman, Phys. Rev. Lett. 106, 130501 (2011)

[8] V. Ansari, E. Roccia, M. Santandrea, M. Doostdar, C. Eigner, L. Padberg, I. Gianani, M. Sbroscia, J. M. Donohue, L. Mancino, M. Barbieri, and C. Silberhorn, Opt. Express 26, 2764 (2018).

[9] B. Brecht, D. V. Reddy, C. Silberhorn, and M. G. Raymer, Phys. Rev. X 5, 041017 (2015).

[10] P. J. Mosley, J. S. Lundeen, B. J. Smith, P. Wasylczyk, A. B. U'Ren, C. Silberhorn, and I. A. Walmsley, Phys. Rev. Lett. 100, 133601 (2008).
[11] S. Carrasco, A. V. Sergienko, B. E. A. Saleh, M. C. Teich, J. P. Torres, and L. Torner, Phys. Rev. A 73, 063802 (2006).

[12] B. J. Smith, P. Mahou, O. Cohen, J. S. Lundeen, and I. A. Walmsley, Opt. Express 17, 23589 (2009).

[13] M. Avenhaus, A. Eckstein, P. J. Mosley, and C. Silberhorn, Opt. Lett. 34, 2873 (2009).

[14] I. Jizan, L. G. Helt, C. Xiong, M. J. Collins, D. Choi, C. J. Chae, M. Liscidini, M. J. Steel, B. J. Eggleton, and A. S. Clark, Sci. Rep. 5, 12557 (2015).

[15] J.-P. W. MacLean, J. M. Donohue, and K. J. Resch, Phys. Rev. Lett. 120, 053601 (2018).

[16] I. Jizan, B. Bell, L. G. Helt, A. Casas Bedoya, C. Xiong, and B. J. Eggleton, Opt. Lett. 41, 4803 (2016).

[17] K. Park, J. Kim, T. Zhao, Y. Cho, and Y. Kim, Optica 4, 1293 (2017).

[18] I. A. Walmsley and C. Dorrer, Adv. Opt. Photonics 1, 308 (2009).

[19] J.-P. W. MacLean, S. Schwarz, and K. J. Resch, Phys. Rev. A 100, 033834 (2019).

[20] A. O. C. Davis, V. Thiel, M. Karpinski, and B. J. Smith, Phys. Rev. A 98, 023840 (2018).

[21] C. Iaconis and I. A. Walmsley, Opt. Lett. 23, 792 (1998).

[22] E. M. Kosik, A. S. Radunsky, I. A. Walmsley, and C. Dorrer, Opt. Lett. 30, 326 (2005). 
[23] T. M. Shuman, M. E. Anderson, J. Bromage, C. Iaconis, L. Waxer, and I. A. Walmsley, Opt. Express 5, 134 (1999).

[24] P. Baum, S. Lochbrunner, and E. Riedle, Opt. Lett. 29, 210 (2004).

[25] C. Dorrer, P. Londero, and I. A. Walmsley, Opt. Lett. 26, 1510 (2001).

[26] T. Witting, D. R. Austin, and I. A. Walmsley, Opt. Exp. 17, 18983 (2009).

[27] D. R. Austin, T. Witting, and I. A. Walmsley, J. Opt. Soc. Am. 26, 1818 (2009).

[28] D. R. Austin, T. Witting, and I. A. Walmsley, Opt. Lett. 35, 1971 (2010).

[29] C. Bourassin-Bouchet, M. M. Mang, I. Gianani, and I. A. Walmsley, Opt. Lett. 38, 5299 (2013).

[30] I. Gianani, C. Bourassin-Bouchet, P. N. Anderson, M. M. Mang, A. S. Wyatt, M. Barbieri, and I. A. Walmsley, arXiv:1612.06937.
[31] M. M. Mang, C. Bourassin-Bouchet, and I. A. Walmsley, Opt. Lett. 39, 6142 (2014).

[32] P. N. Anderson, D. T. Lloyd, I. Gianani, F. Wiegandt, D. L J. Treacher, M. M. Mang, A. Schiavi, K. O'Keefe, S. M. Hooker, and I. A. Walmsley (unpublished).

[33] A. Cabello, A. Rossi, G. Vallone, F. De Martini, and P. Mataloni, Phys. Rev. Lett. 102, 040401 (2009).

[34] G. Vallone, I. Gianani, E. B. Inostroza, C. Saavedra, G. Lima, A. Cabello, and P. Mataloni, Phys. Rev. A 83, 042105 (2011).

[35] J. D. Franson, Phys. Rev. Lett. 62, 2205 (1989).

[36] C. Dorrer and I. Kang, Opt. Lett. 28, 477 (2003).

[37] C. K. Law, I. A. Walmsley, and J. H. Eberly, Phys. Rev. Lett. 84, 5304 (2000).

[38] M. E. Anderson, L. E. E. de Araujo, E. M. Kosik, and I. A. Walmsley, Appl. Phys. B 70, S85 (2000). 